\title{
PARAMETRIC VERSUS NONPARAMETRIC DATA-AIDED CHANNEL ESTIMATION IN A MULTIPATH FADING ENVIRONMENT
}

\author{
D. Van Welden, M. Moeneclaey and H. Steendam \\ DIGCOM research group, TELIN Department, Ghent University \\ Sint-Pietersnieuwstraat 41, 9000 Gent, Belgium \\ E-mail: $\{$ dmvwelde,mm,hs\}@ telin.ugent.be
}

\begin{abstract}
This paper compares a parametric and a nonparametric channel estimation method in a multipath fading environment. In the parametric method the gains and delays are estimated and these estimates are used to compute the samples of the received symbol pulse. In the nonparametric method the samples of the received pulse are estimated directly, ignoring the underlying multipath channel structure. We compare these two channel estimation methods in terms of accuracy (characterized by the MSE on the samples of the received pulse) and computational complexity. We also investigate the influence of channel estimation errors on the BER of a decision feedback equalizer.
\end{abstract}

\section{INTRODUCTION}

When transmitting data over multipath fading channels, the receiver needs channel state information (CSI) in order to detect the transmitted symbols. CSI can be acquired by means of a parametric or a nonparametric channel estimation method. In the parametric method the gains and delays of the different paths are estimated, and these estimates are used to compute an estimate of the samples of the received symbol pulse. In the nonparametric method the samples of the received symbol pulse are estimated directly, ignoring the underlying structure imposed by the propagation paths.

In this paper we compare these two channel estimation methods in a data-aided scenario. For the parametric method, we consider the iterative space-alternating generalized expectation-maximization (SAGE) algorithm as proposed in [1], because the maximum likelihood (ML) estimator is too complex. For the nonparametric method, ML estimation is used [2]. For both estimation methods, we simulate the mean-square error (MSE) on the estimates of the sampled received pulse, and analytically derive the respective Cramer Rao Bound (CRB) on this MSE. Further, the influence of estimation errors on the bit error rate (BER) is also investigated by considering the signal-to-interference-plus-noise ratio (SINR) at the output of a fractionally spaced decision feedback equalizer (DFE) [3], [4] that is computed from the estimated received pulse. Finally the computational complexity of both methods is discussed.

\section{SySTEM MODEL}

Consider the transmission of a linearly modulated signal with symbol period $T$ over a slowly fading multipath channel with $L$ distinct paths. The received symbol pulse $h(t)$ is given by

$$
h(t)=\sum_{l=0}^{L-1} \alpha_{l} p\left(t-\tau_{l}\right)
$$

where $p(t)$ is the transmitted symbol pulse and $\alpha_{l}$ and $\tau_{l}$ denote the complex gain and the path delay of the $l$-th propagation path. In order to facilitate channel estimation, a pilot sequence $\{b(k), k=0, \ldots, K-1\}$ is transmitted at regular intervals. We assume that the channel is time-invariant over the duration between successive pilot sequences. The received signal is sampled at a sampling frequency of $1 / T_{s}$. The samples $r\left(m T_{s}\right)$ of the received signal are given by

$$
r\left(m T_{S}\right)=\sum_{k} b(k) h\left(m T_{s}-k T\right)+w\left(m T_{S}\right)
$$

where $w\left(m T_{s}\right)$ is complex-valued white Gaussian noise with variance $N_{0} / T_{s}$, added by the channel.

In order to compare the estimator performance of both channel estimation methods, we consider the following meansquare error on the samples of the received symbol pulse:

$$
M S E=\mathrm{E}\left[\sum_{m}\left|\hat{h}\left(m T_{s}\right)-h\left(m T_{s}\right)\right|^{2}\right]
$$

where $\hat{h}\left(m T_{s}\right)$ are the estimated samples of the received symbol pulse.

\section{PARAmetric Channel estimation}

The parametric method involves the estimation of the path parameters $\left\{\alpha_{l}, \tau_{l}, l=0, \ldots, L-1\right\}$. It can be verified that ML estimation yields an $L$-dimensional search to estimate the $L$ path delays, after which the $L$ path gain estimates are obtained analytically [5]. In order to avoid the high computational complexity of the $L$-dimensional search, we resort to the iterative SAGE algorithm [1]. Each SAGE iteration involves $L$ steps: in the $l$-th step of the $i$-th iteration, we perform a 1-dimensional search to estimate $\tau_{l}$ and an analytic computation to estimate $\alpha_{l}$, by using the most recent estimates of the gains and the delays of the other paths for subtracting their estimated path interference from the received 
signal. Denoting the resulting path parameter estimates as $\left\{\hat{\alpha}_{l}, \hat{\tau}_{l}, l=0, \ldots, L-1\right\}$, the estimates of the samples of the received symbol pulse $h(t)$ are computed as

$$
\hat{h}\left(m T_{s}\right)=\sum_{l=0}^{L-1} \hat{\alpha}_{l} p\left(m T_{s}-\hat{\tau}_{l}\right)
$$

\section{A. Cramer Rao bound}

We define $\delta(m)$ as the estimation error on $h\left(m T_{S}\right)$ :

$$
\delta(m)=\sum_{l=0}^{L-1} \hat{\alpha}_{l} p\left(m T_{s}-\hat{\tau}_{l}\right)-\sum_{l=0}^{L-1} \alpha_{l} p\left(m T_{s}-\tau_{l}\right)
$$

For small estimation errors on $\left\{\alpha_{l}, \tau_{l}, l=0, \ldots, L-1\right\}$, $\delta(m)$ can be approximated as

$\delta(m) \simeq \sum_{l=0}^{L-1}\left(\Delta \alpha_{l \Re}+j \Delta \alpha_{l \mathfrak{I}}\right) p\left(m T_{s}-\tau_{l}\right)-\sum_{l=0}^{L-1} \alpha_{l} \Delta \tau_{l} \dot{p}\left(m T_{s}-\tau_{l}\right)$

where $\Delta \alpha_{l \Re}+j \Delta \alpha_{l \mathfrak{I}}=\hat{\alpha}_{l}-\alpha_{l}$ and $\Delta \tau_{l}=\hat{\tau}_{l}-\tau_{l}$. This expression can be written in a more compact form by using vector notation:

$$
\delta(m)=\mathbf{p}_{m}^{T} \varepsilon
$$

where $\varepsilon=\left[\Delta \alpha_{0 \Re}, \ldots, \Delta \alpha_{L-1 \Re}, \Delta \alpha_{0 \mathfrak{I}}, \ldots, \Delta \alpha_{L-1 \mathfrak{I}}, \Delta \tau_{0}, \ldots\right.$, $\left.\Delta \tau_{L-1}\right]^{T}$ and $\mathbf{p}(m)=\left[p\left(m T_{s}-\tau_{0}\right), \ldots, p\left(m T_{s}-\tau_{L-1}\right)\right.$, $j p\left(m T_{s}-\tau_{0}\right), \ldots, j p\left(m T_{s}-\tau_{L-1}\right),-\alpha_{0} \dot{p}\left(m T_{s}-\tau_{0}\right), \ldots$, $\left.-\alpha_{L-1} \dot{p}\left(m T_{S}-\tau_{L-1}\right)\right]^{T}$. Using (7), the MSE (3) can be written as:

$$
M S E=\sum_{m} \mathbf{p}_{m}^{T} \mathrm{E}\left[\varepsilon \varepsilon^{H}\right] \mathbf{p}_{m}^{*}
$$

This MSE can be lower bounded by the CRB:

$$
\sum_{m} \mathbf{p}_{m}^{T} \mathrm{E}\left[\varepsilon \varepsilon^{H}\right] \mathbf{p}_{m}^{*} \geq \sum_{m} \mathbf{p}_{m}^{T} \mathbf{J}_{p}^{-1} \mathbf{p}_{m}^{*}
$$

where $\mathbf{J}_{p}$ denotes the Fischer information matrix related to the estimation of $\left\{\alpha_{l}, \tau_{l}, l=0, \ldots, L-1\right\}$ [6].

\section{B. Computational complexity}

The parametric channel estimation method can be divided in three parts: A first part involves applying the received signal to a filter matched to the transmitted pilot signal, and computing the matched filter output at $N_{\tau}$ instants with spacing $\Delta$, such that $N_{\tau} \Delta$ covers the uncertainty region of the path delays. A second parts consists of the different iterations of the SAGE algorithm for estimating the path gains and delays. The third part is the computation of the estimated samples of the received symbol pulse from the gain and delay estimates.

Denoting by $K$ the number of pilot symbols, $1 / T_{s}$ the sampling rate at the receiver, $N_{p} T_{s}$ the duration of the transmit pulse $p(t), N_{i t}$ the number of iterations performed in the SAGE algorithm, and $N$ the number of estimated samples of the received pulse, the number of multiplications (of complex numbers) required by the different phases is shown in Table I.

\section{NONPARAMETRIC CHANNEL ESTIMATION}

The nonparametric method directly estimates the samples $\left\{h\left(m T_{S}\right)\right\}$ from the received signal, without exploiting the relation (1). ML estimation [2] involves the multiplication of a pilot-dependent matrix and the vector of received signal samples. This yields

$$
\hat{\mathbf{h}}=\left(\mathbf{B}^{H} \mathbf{B}\right)^{-1} \mathbf{B}^{H} \mathbf{r}
$$

where $\mathbf{B}$ is a pilot-dependent matrix and $\mathbf{r}$ is the vector of received signal samples.

\section{A. Cramer Rao bound}

It can be shown that the Fisher information matrix $\mathbf{J}_{n p}$ of $\{\mathfrak{R}(\mathbf{h}), \mathfrak{I}(\mathbf{h})\}$ is given by [6]

$$
\mathbf{J}_{n p}=\frac{2 T_{s}}{N_{0}}\left[\begin{array}{rr}
\mathfrak{R}\left(\mathbf{B}^{H} \mathbf{B}\right) & -\mathfrak{I}\left(\mathbf{B}^{H} \mathbf{B}\right) \\
\mathfrak{I}\left(\mathbf{B}^{H} \mathbf{B}\right) & \mathfrak{R}\left(\mathbf{B}^{H} \mathbf{B}\right)
\end{array}\right]
$$

which yields the following CRB:

$$
M S E=\mathrm{E}\left[\sum_{m}\left|\hat{h}\left(m T_{s}\right)-h\left(m T_{s}\right)\right|^{2}\right] \leq \operatorname{tr}\left(\mathbf{J}_{n p}^{-1}\right)
$$

where $\operatorname{tr}(\mathbf{X})$ denotes the trace of $\mathbf{X}$.

\section{B. Computational complexity}

The computational complexity of the nonparametric channel estimation method is determined by the computational complexity of the multiplication of the pilot-dependent matrix $\left(\mathbf{B}^{H} \mathbf{B}\right)^{-1} \mathbf{B}^{H}$ with dimensions $\left(N_{s}(K-1)+N\right) \times N$ and the vector of received signal samples $\mathbf{y}$ because the matrix $\left(\mathbf{B}^{H} \mathbf{B}\right)^{-1} \mathbf{B}^{H}$ can be stored at the receiver. This results in a total number

$$
N_{\text {mult }, n p}=(K-1) N+\frac{N^{2}-1}{N_{s}}+1
$$

of complex multiplications for the nonparametric channel estimation method. $N$ can be substituted by $N_{S} D_{T}+1$ in (13) yielding

$$
N_{\text {mult }, n p}=(K-1) N_{S} D_{T}+N_{s} D_{T}^{2}+2 D_{T}+K
$$

where $D_{T}$ denotes the duration of the received pulse expressed in symbol periods. 


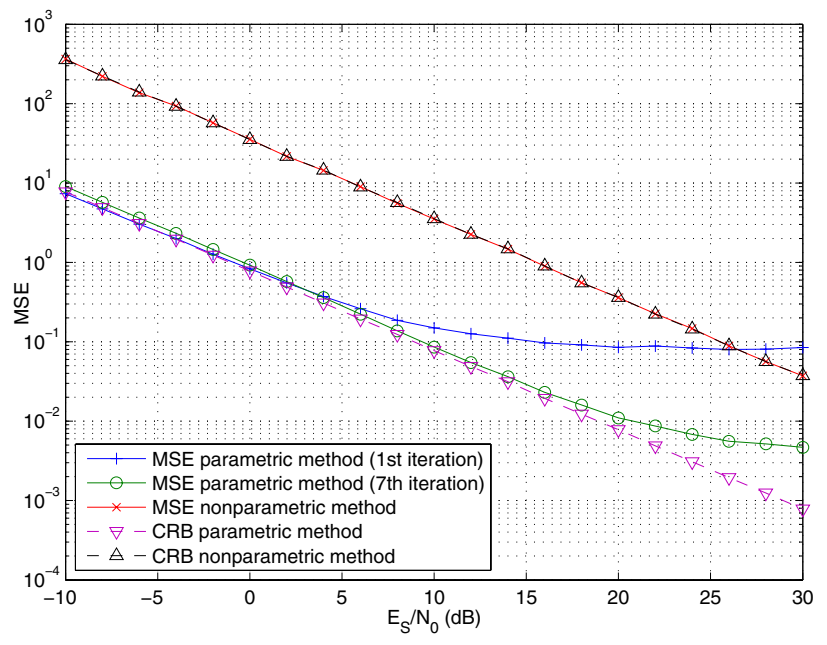

Fig. 1. Comparison between the two estimation methods in terms of the MSE

\section{SINR AT THE OUTPUT OF A FRACTIONALLY SPACED DFE}

In this contribution we consider a fractionally spaced MMSE DFE [4] as receiver. The DFE consists of a forward filter $\mathbf{h}_{\mathbf{e q}}$ which has $K_{F F 1}$ anti-causal coefficients and $1+K_{F F 2}$ causal coefficients, and a feedback filter $\mathbf{h}_{\mathbf{F B}}$ which has $K_{F B}$ coefficients. We denote the transmitted data symbols also as $b(k)$. The input $u(k)$ to the symbol-by-symbol detector is

$$
u(k)=\sum_{i=-K_{F F 1}}^{K_{F F 2}} h_{e q}(i) r\left(k N_{s}-i\right)-\sum_{m=1}^{K_{F B}} h_{F B}(m) \hat{b}(k-m)
$$

where $\hat{b}(k-m)$ are the past decisions and $r\left(k T_{s}\right)$ is the received signal (2) but with $\{b(k)\}$ now denoting the unknown data symbols instead of pilot symbols. The equalizer coefficients $\mathbf{h}_{\mathbf{e q}}$ and $\mathbf{h}_{\mathbf{F B}}$ are selected such that $\mathrm{E}\left[|u(k)-b(k)|^{2}\right]$ is minimized (under the assumption that past decisions are correct). In order to compute the optimum equalizer coefficients, the samples $\left\{h\left(m T_{s}\right)\right\}$ of the received symbol pulse are needed. As these samples are not known to the receiver, the estimated samples $\left\{\hat{h}\left(m T_{s}\right)\right\}$ are used instead, yielding suboptimum equalizer coefficients.

The equalizer output $u(k)$ from (15) consists of a useful term (proportional to $b(k)$ ), a residual ISI term (linear combination of symbols $b(m)$ with $m \neq k$ ) and an additive noise term. As a performance measure we consider the SINR at the equalizer output, which is defined as the ratio of the power of the useful term to the power of the residual ISI plus noise.

\section{Simulation Results}

The transmit pulse is a square root raised cosine pulse with $25 \%$ roll-off, truncated to a length of 20 symbol intervals. The pilot sequence consists of 10 BPSK symbols. We consider a multipath fading channel with 2 paths. We assume that $\tau_{0}$ is uniformly distributed in $(0, T)$, the delay difference $\tau_{1}-\tau_{0}$ is uniformly distributed in $(0,2 T)$. The channel gains $\alpha_{l}$ are

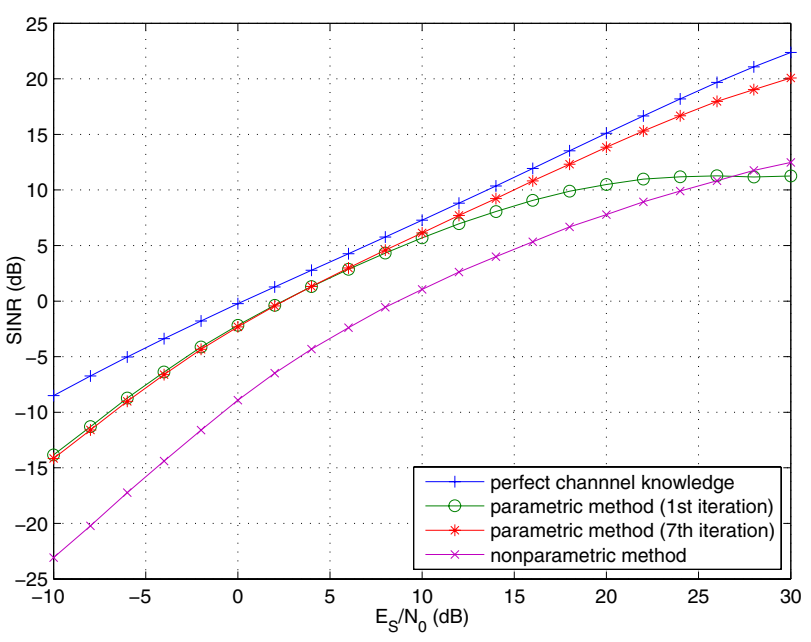

Fig. 2. Comparison between the two estimation methods in terms of the SINR at the DFE output

complex-valued Gaussian random variables with zero mean and variance $1 / 2$ (Rayleigh fading). The sample period $T_{s}$ is set to $T / 2$ so that no aliasing occurs. Taking into account the duration $(20 T)$ of the transmit pulse, the uncertainty $(T)$ on $\tau_{0}$ and the delay spread $(2 T)$ of the channel, the received pulse $h(t)$ has $N=47$ samples taken at rate $1 / T_{s}=2 / T$. Hence, the parametric method involves the estimation of 2 delays and 2 complex gains, while the nonparametric method consists of estimating 47 samples $h\left(m T_{s}\right)$. The parametric method uses $N_{\tau}=31$ and $\Delta=T / 10$.

For the two estimation methods, Figure 1 shows, as a function of $E_{s} / N_{0}$, the MSE (3) averaged over different realizations of the channel and of the pilot sequence along with their respective CRB. We observe that the parametric method yields the smaller CRB; this is because the parametric method exploits the underlying multipath channel model. The MSE resulting from the nonparametric method essentially coincides with the corresponding CRB. After a sufficient number of iterations, the MSE resulting from the parametric method is close to the corresponding CRB at small and moderate $E_{s} / N_{0}$, but exhibits an error floor at high $E_{S} / N_{0}$. This floor is caused by the discretization of the unknown delays when performing the 1-dimensional searches associated with the SAGE algorithm (we have verified that a finer discretization indeed lowers the floor).

In Figure 2 the SINR at the output of a $T / 2$-spaced DFE, with $K_{F F 1}=16$ anti-causal forward coefficients, $1+$ $K_{F F 2}=17$ causal forward coefficients and $K_{F B}=1$ feedback coefficient, is shown in order to illustrate the effect of channel estimation errors on the reliability of the communication link. The equalizer coefficients are computed from the estimated samples of the received symbol pulse. We observe that the SINR associated with the parametric channel estimation method is approximately $1 \mathrm{~dB}$ below the SINR corresponding to perfect channel estimates for moderate $E_{S} / N_{0}$, whereas the 
SINR resulting from the nonparametric method is about $5 \mathrm{~dB}$ less than the SINR corresponding to the parametric method. Hence for the example considered, the nonparametric method yields an SINR degradation of $4 \mathrm{~dB}$ as compared to the parametric method.

Let us now discuss the computational complexity. For the parametric method the example mentioned here results in 3225 complex multiplications when 7 SAGE iterations are carried out. 1860 complex multiplications or $60 \%$ of the total amount is needed for the first part of the algorithm. For the nonparametric method the required number of complex multiplications for the considered situation equals 1528. Hence the parametric method needs 2 times as much multiplications as the nonparametric method.

\section{CONCLUSION AND REMARKS}

In this contribution we have compared parametric and nonparametric channel estimation from pilot symbols. We derived analytically the Cramer-Rao lowerbound on the MSE of the samples of the received symbol pulse for both methods. For $E_{s} / N_{0}$ values of practical interest, we have shown that the former method outperforms the latter method, both in terms of estimation accuracy and detector performance. However, the nonparametric channel estimation method has a considerably lower computational complexity than the parametric channel estimation method.

Our numerical example involves two Rayleigh fading paths of equal average power with uniformly distributed path delays, but the parametric and nonparametric estimation methods can equally be applied for another number of paths, other fading statistics, other power delay profiles and other path delay distributions. Also in these cases, the parametric method outperforms the nonparametric method, because the former takes advantage of the multipath structure of the channel.

\section{REFERENCES}

[1] B. Fleury, M. Tschudin, R. Heddergott, D. Dahlhaus, and K. Pedersen. Channel Parameter Estimation in Mobile Radio Environments Using the SAGE Algorithm. IEEE Journal on Selected Areas in Communications, 17(3):434-450, March 1999.

[2] H. Meyr, M. Moeneclaey, S.A. Fechtel. Synchronization, Channel Estimation, and Signal Processing, volume 2 of Digital Communication Receivers. John Wiley \& Sons, 1997.

[3] R.D. Gitlin, J.F. Hayes and S.B. Weinstein. Data Communications Principles. Plenum Press, 1992.

[4] S.U.H. Qureshi. "Adaptive Equalization". Proceedings of the IEEE, 73(9):1349-1387, September 1985.

[5] E.G. Strom and F. Malmsten. A maximum likelihood approach for estimating DS-CDMA multipath fading channels. IEEE Journal on Selected Areas in Communications, 18(1):132-140, January 2000.

[6] H.L. Van Trees. Detection, Estimation, and Modulation Theory, Part I. Wiley and Sons, October 2001. 